Supporting Information of

\title{
Auxetic Carbon Honeycomb: Strain-Tunable Phase Transitions and novel Negative Poisson's Ratio
}

Yanchun Li, Shuaiwei Wang *, Baocheng Yang *

Henan Provincial Key Laboratory of Nanocomposites and Applications, Institute of Nanostructured Functional Materials, Huanghe Science and Technology College, Zhengzhou 450006, Henan, China

*To whom correspondence should be addressed. Email: shuaiweiwang@hotmail.com(S.W.) baochengyang@yahoo.cn(B.Y.) 


\section{Section I. Computation details}

Vienna ab initio simulation package (VASP) ${ }^{1-2}$ is employed to perform the first principle calculations. The ion-electron interactions were described by projectoraugmented plane wave (PAW) $)^{3}$ potential. The electron exchange-correlation energy was treated by the generalized gradient approximation (GGA) with Perdew-BurleErnzerhof (PBE) ${ }^{4}$ functional. The energy cutoff of plane waves was set to $600 \mathrm{eV}$ and the energy convergence criterion between two steps was set at $10^{-8} \mathrm{eV}$. The atomic structures were fully relaxed using the conjugate gradients (CG) method until the maximum force on each atom was less than $10^{-2} \mathrm{eV} \AA^{-1}$. The Brillouin zone (BZ) was sampled with a spacing of $2 \pi \times 0.02 \AA^{-1}$. The atomic positions can be fully relaxed under the condition of tensile strain. The lattice constants are fixed in the tensile strain direction, while no limit in the other directions.

\section{Section II. Validation of the theoretical calculations}

We firstly computed the basic properties of CHCs and compared with other carbon materials (e.g. diamond, Bco-C16, Bct-C40, Bct-C 16 , and Hex- $\mathrm{C}_{18}$ ). The results show that the first-principles calculations are able to accurately reproduce the lattice constant and total energy of graphene and other 3D carbon materials in comparison with experimental results ${ }^{5-7}$ and/or DFT calculations ${ }^{8-14}$ from other research groups (See Table S2). Regarding the mechanical properties for graphene, the stress-strain curves (See Figure S3) can be well matched with Wei et al. results ${ }^{15}$. 
Table S1. The space group, lattice constants $(\AA)$, bond lengths $(\AA)$, and cohesive energy $E_{\text {coh }}$ (eV/atom) of optimized Cmcm-CHC, Cmmm-CHC, $\mathrm{P}_{3} / \mathrm{mmc}-\mathrm{CHC}$, and $\mathrm{P} 6 / \mathrm{mmm}-$ CHC-2.

\begin{tabular}{|c|c|c|c|c|c|c|}
\hline \multirow{2}{*}{ Structure } & \multirow{2}{*}{$\begin{array}{l}\text { Space } \\
\text { group }\end{array}$} & \multicolumn{3}{|c|}{ Lattice constants } & \multirow{2}{*}{ bond lengths } & \multirow{2}{*}{$E_{\text {coh }}$} \\
\hline & & $a$ & $b$ & $c$ & & \\
\hline Cmcm-CHC & $\mathrm{Cmcm}$ & 5.93 & 6.31 & 2.47 & $1.53,1.52,1.41$ & $\begin{array}{c}- \\
7.65\end{array}$ \\
\hline $\mathrm{Cmmm}-\mathrm{CHC}$ & $\mathrm{Cmmm}$ & 13.60 & 8.98 & 2.47 & $1.34,1.49,1.53,1.42,1.45$ & $\begin{array}{c}- \\
7.68\end{array}$ \\
\hline $\mathrm{P}_{3} / m m c-\mathrm{CHC}$ & $\mathrm{P}_{3} / m m c$ & 4.46 & 4.46 & 2.53 & $1.53,1.50$ & $\begin{array}{c}- \\
7.53\end{array}$ \\
\hline P6/mmm-CHC & P6/mmm & 8.13 & 8.13 & 2.51 & $1.56,1.47,1.36$ & $\begin{array}{c}- \\
7.61\end{array}$ \\
\hline
\end{tabular}

Table S2. Calculated lattice parameters and total energy of Diamond, Bco-C16, Bct$\mathrm{C} 40$, Bct- $\mathrm{C}_{16}$, and Hex- $\mathrm{C}_{18}$, together with DFT and experimental data from other research groups.

\begin{tabular}{|c|c|c|c|c|c|c|}
\hline Structure & Method & $\mathrm{a}(\AA)$ & $\mathrm{b}(\AA)$ & $\mathrm{c}(\AA)$ & Total energy $(\mathrm{eV})$ & Reference(s) \\
\hline Cmcm-CHC & PBE & 5.93 & 6.31 & 2.47 & -8.98 & This work \\
\hline Cmmm-CHC & PBE & 13.60 & 8.98 & 2.47 & -9.01 & This work \\
\hline \multirow{3}{*}{ Diamond } & AM05 & 3.552 & 3.552 & 3.552 & -9.02 & This work \\
\hline & PBE & 3.572 & 3.572 & 3.572 & -9.10 & This work \\
\hline & Exp & 3.567 & 3.567 & 3.567 & & 7 \\
\hline \multirow{3}{*}{ Graphite } & PBE & 2.463 & 2.463 & 6.856 & -9.22 & This work \\
\hline & AM05 & 2.457 & 2.457 & 6.784 & -9.05 & This work \\
\hline & Exp & 2.460 & 2.460 & 6.704 & & 7 \\
\hline \multirow{3}{*}{ Bco-C16 } & AM05 & 7.802 & 4.883 & 3.235 & -8.66 & This work \\
\hline & PBE & 7.803 & 4.897 & 3.299 & -8.82 & This work \\
\hline & AM05 & 7.806 & 4.877 & 3.237 & -8.67 & 16 \\
\hline \multirow{3}{*}{ Bct-C40 } & AM05 & 12.883 & 12.883 & 2.47 & -8.90 & This work \\
\hline & PBE & 12.947 & 12.947 & 2.47 & -9.04 & This work \\
\hline & AM05 & 12.901 & 12.901 & 2.46 & -8.91 & 13 \\
\hline \multirow{2}{*}{ Bct- $\mathrm{C}_{16}$} & PBE & 6.591 & 6.591 & 3.386 & -8.54 & This work \\
\hline & PBE & 6.592 & 6.592 & 3.377 & -8.54 & 17 \\
\hline \multirow{2}{*}{ Hex $-\mathrm{C}_{18}$} & PBE & 8.33 & 8.33 & 2.46 & -7.93 & This work \\
\hline & PBE & 8.36 & 8.36 & 2.46 & & 14 \\
\hline \multirow{3}{*}{ graphene } & PBE & 2.468 & & & -9.23 & This work \\
\hline & PBE & 2.468 & & & -9.23 & $9-10$ \\
\hline & Exp & 2.45 & & & & 5 \\
\hline
\end{tabular}


Figure.S1. The Poisson's ratio as a function of uniaxial deformation of $\mathrm{Cmcm}$-CHC structure. (a) strain $\varepsilon_{y}$ versus $\varepsilon_{x}$ and $\varepsilon_{z}$ for $\mathrm{Cmcm}$-CHC structure subjected to uniaxial tensile loading in the zigzag direction. (b) the poisson's ratio of $\mathrm{Cmcm}$-CHC structure in $x$ and $z$ axis. (c) strain $\varepsilon_{z}$ versus $\varepsilon_{y}$ and $\varepsilon_{x}$ for $C m m m$-CHC structure subjected to uniaxial tensile loading in the tube axis. (d) the poisson's ratio of $\mathrm{Cmmm}-\mathrm{CHC}$ structure in $x$ and $y$ axis.
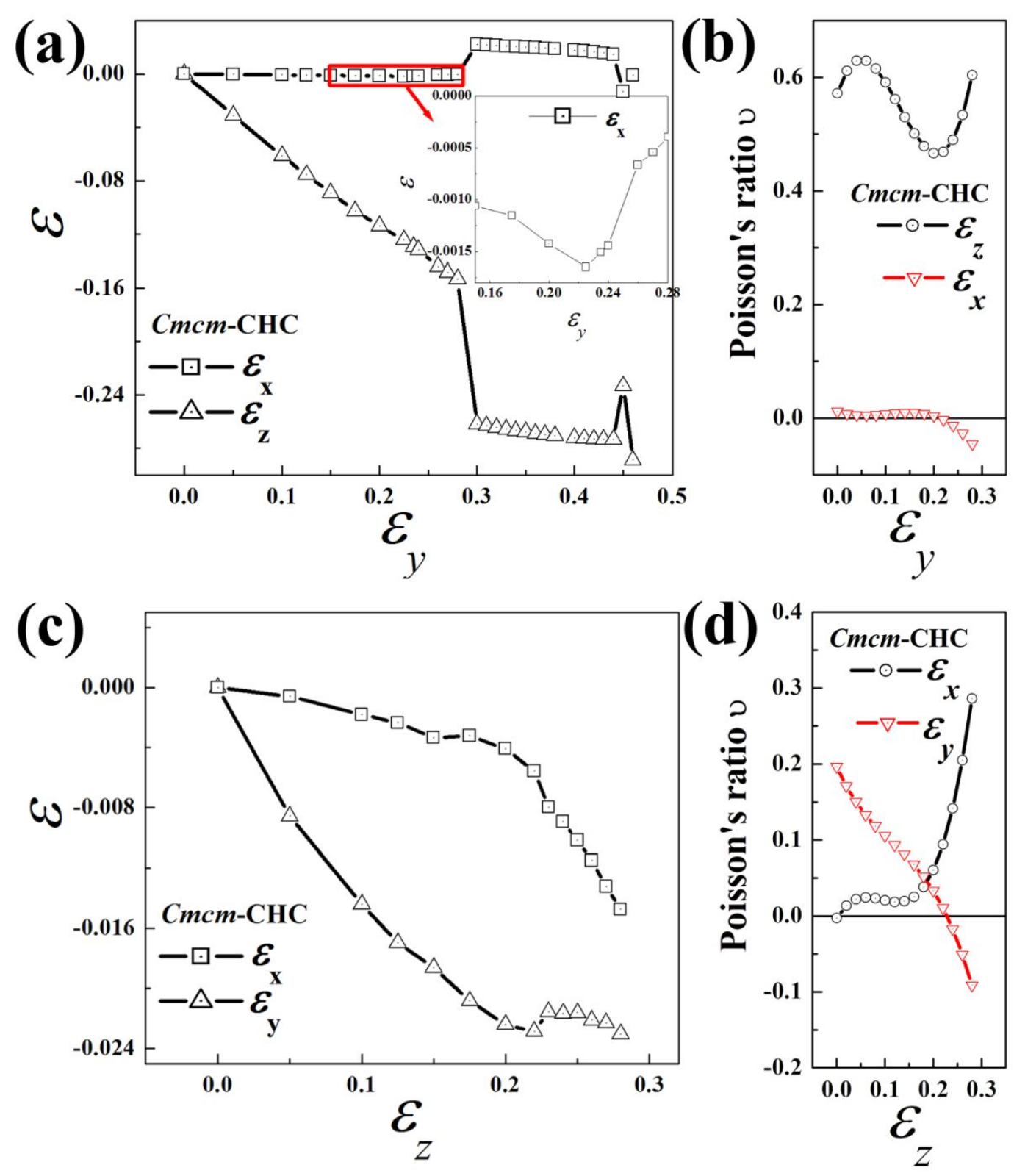
Figure.S2. The phonon dispersion of $\mathrm{P}_{3} / \mathrm{mmc}-\mathrm{CHC}(\mathrm{a}-\mathrm{b})$ and $\mathrm{P} 6 / \mathrm{mmm}-\mathrm{CHC}(\mathrm{c}-\mathrm{d})$ at the different strain.

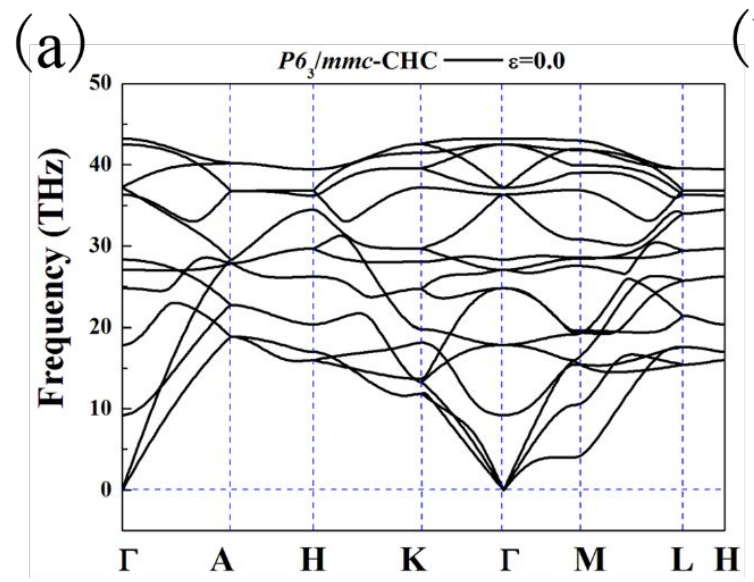

(b)
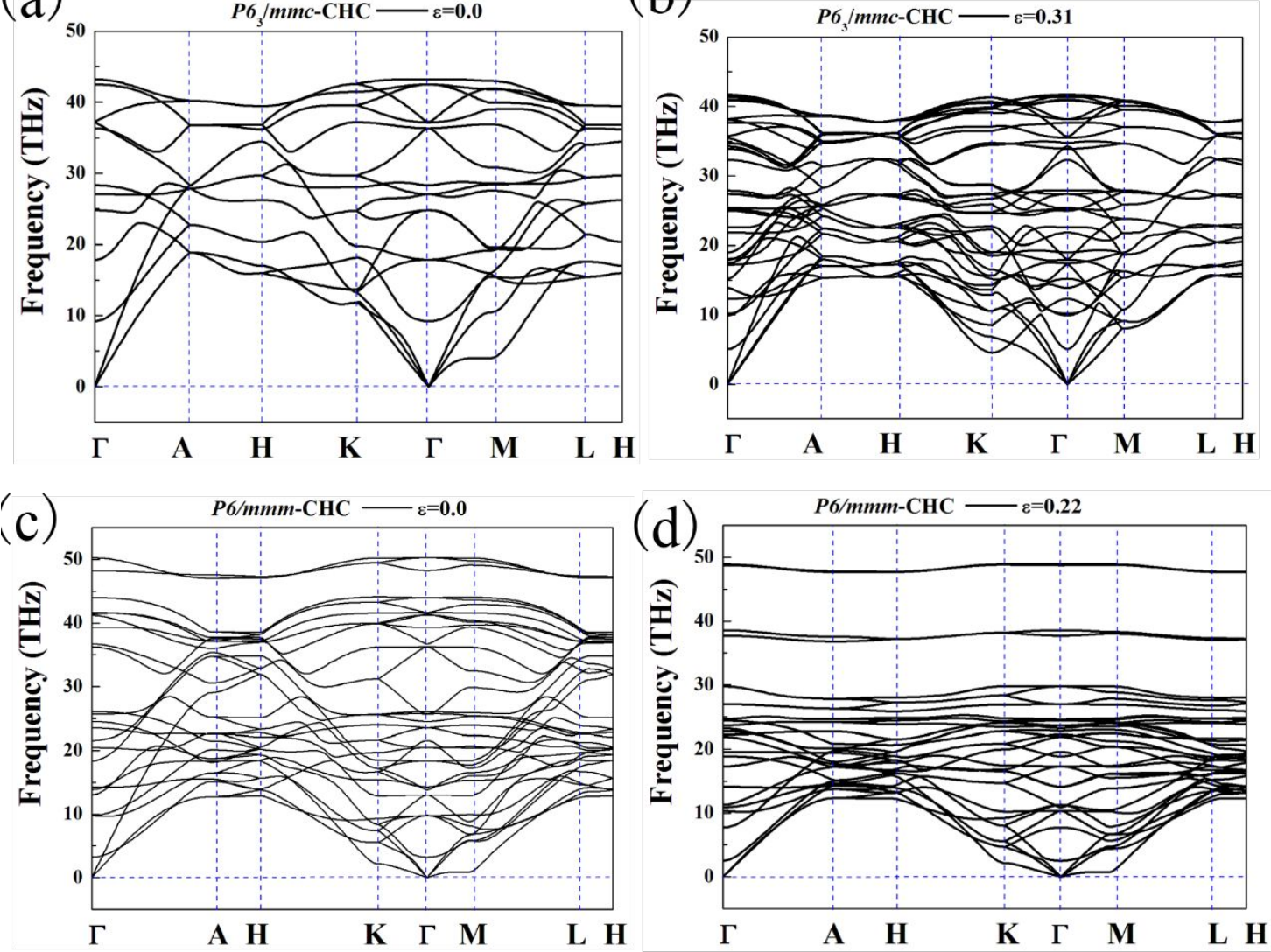
Figure S3. The stress-strain curves of graphene under tensile loading along the armchair and zigzag directions and comparison with other DFT results.

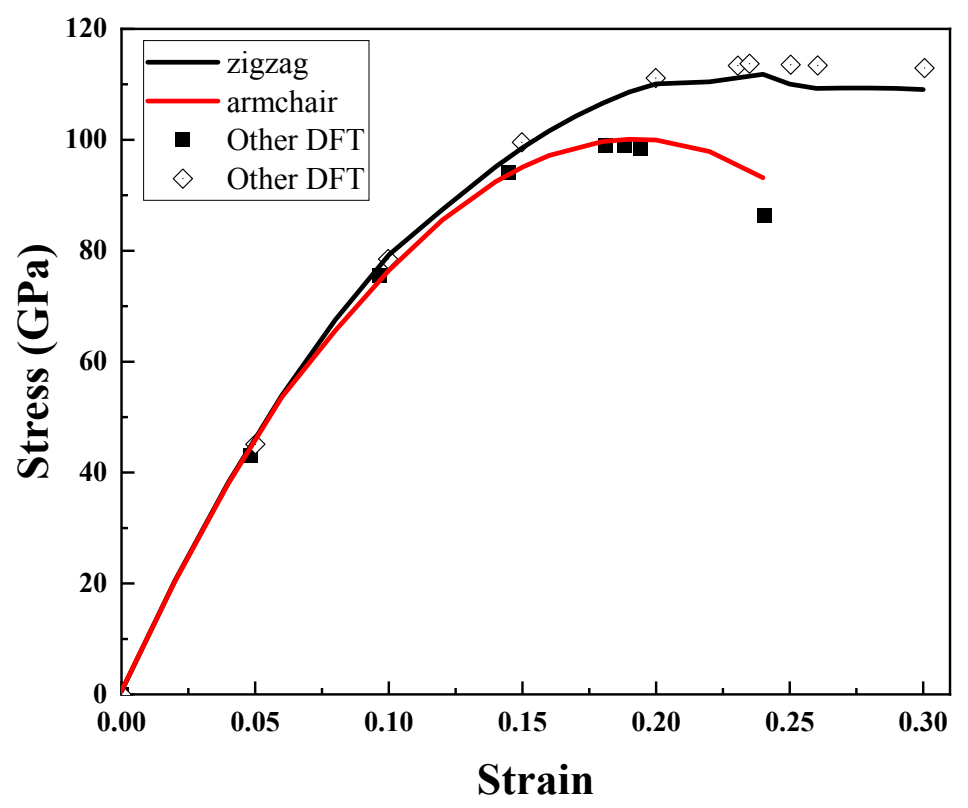




\section{References}

(1) Kresse, G.; Hafner, J., Ab initio molecular dynamics for open-shell transition metals. Phys. Rev. B 1993, 48, 13115.

(2) Kresse, G.; Furthmüller, J., Efficient iterative schemes for ab initio total-energy calculations using a plane-wave basis set. Phys. Rev. B 1996, 54, 11169.

(3) Kresse, G.; Joubert, D., From ultrasoft pseudopotentials to the projector augmented-wave method. Phys. Rev. B 1999, 59, 1758.

(4) Perdew, J. P.; Burke, K.; Ernzerhof, M., Generalized gradient approximation made simple. Phys. Rev. Lett. 1996, 77, 3865.

(5) Warner, J. H.; Margine, E. R.; Mukai, M.; Robertson, A. W.; Giustino, F.; Kirkland, A. I., Dislocation-Driven Deformations in Graphene. Science 2012, 337, 209-212.

(6) Castro Neto, A. H.; Guinea, F.; Peres, N. M. R.; Novoselov, K. S.; Geim, A. K., The electronic properties of graphene. Rev. Mod. Phys. 2009, 81, 109-162.

(7) Occelli, F.; Loubeyre, P.; LeToullec, R., Properties of diamond under hydrostatic pressures up to $140 \mathrm{GPa}$. Nat. Mater. 2003, 2, 151-154.

(8) Șahin, H.; Cahangirov, S.; Topsakal, M.; Bekaroglu, E.; Akturk, E.; Senger, R. T.; Ciraci, S., Monolayer honeycomb structures of group-IV elements and III-V binary compounds: Firstprinciples calculations. Phys. Rev. B 2009, 80, 155453.

(9) Lu, H.; Li, S.-D., Two-dimensional carbon allotropes from graphene to graphyne. J. Mater. Chem. C 2013, 1, 3677-3680.

(10) Wang, Z.; Zhou, X.-F.; Zhang, X.; Zhu, Q.; Dong, H.; Zhao, M.; Oganov, A. R., Phagraphene: a low-energy graphene allotrope composed of 5-6-7 carbon rings with distorted 
dirac cones. Nano Lett. 2015, 15, 6182-6186.

(11) Boukhvalov, D. W.; Katsnelson, M. I., Chemical functionalization of graphene. J. Phys.: Condens. Matter. 2009, 21, 344205.

(12) Wang, J.; Weng, H.; Nie, S.; Fang, Z.; Kawazoe, Y.; Chen, C., Body-Centered Orthorhombic $\mathrm{C}_{16}$ : A Novel Topological Node-Line Semimetal. Phys. Rev. Lett. 2016, 116, 195501.

(13) Wang, J.; Nie, S.; Weng, H.; Kawazoe, Y.; Chen, C., Topological Nodal-Net Semimetal in a Graphene Network Structure. Phys. Rev. Lett. 2018, 120, 026402.

(14) Liu, J.; Zhao, T.; Zhang, S.; Wang, Q., A new metallic carbon allotrope with high stability and potential for lithium ion battery anode material. Nano Energy 2017, 38, 263-270.

(15) Wei, Y.; Wu, J.; Yin, H.; Shi, X.; Yang, R.; Dresselhaus, M., The nature of strength enhancement and weakening by pentagon-heptagon defects in graphene. Nat. Mater. 2012, $11,759-763$.

(16) Wang, J.; Weng, H.; Nie, S.; Fang, Z.; Kawazoe, Y.; Chen, C., Body-Centered Orthorhombic $\mathrm{C}_{16}$ : A Novel Topological Node-Line Semimetal. Phys. Rev. Lett. 2016, 116, 195501.

(17) Cheng, Y.; Feng, X.; Cao, X.; Wen, B.; Wang, Q.; Kawazoe, Y.; Jena, P., Body-Centered Tetragonal C16: A Novel Topological Node-Line Semimetallic Carbon Composed of Tetrarings. Smal/2017, 13, 1602894. 